\title{
Soliton solution of generalized Zakharov-Kuznetsov and Zakharov-Kuznetsov-Benjamin-Bona-Mahony equations with conformable temporal evolution
}

\author{
H. Rezazadeh ${ }^{a}$, A. Korkmaz ${ }^{b}$, N. Raza ${ }^{c}$, K. K. Ali ${ }^{d}$, and M. Eslami ${ }^{e}$ \\ ${ }^{a}$ Faculty of Engineering Technology, Amol University of Special Modern Technologies, Amol, Iran. \\ e-mail: rezazadehadi1363@gmail.com \\ ${ }^{b}$ The IJEMAPS, Marcel-Paul Str. 146, 99427, Weimar, Germany. \\ e-mail: alperkorkmaz7@gmail.com \\ ${ }^{c}$ Department of Mathematics, University of the Punjab, Quaid-e-Azam Campus, Lahore,Pakestan. \\ e-mail: nauman.math@pu.edu.pk \\ ${ }^{d}$ Mathematics Department, Faculty of Science, Al-Azhar University, Nasr-City, Cairo, Egypt. \\ e-mail: khalidkaram2012@yahoo.com \\ ${ }^{e}$ Department of Mathematics, Faculty of Mathematical Sciences, University of Mazandaran, Babolsar, Iran. \\ e-mail: mostafa.eslami@umz.ac.ir
}

Received 17 November 2020; accepted 4 January 2021

\begin{abstract}
In this paper, we propose the method of functional variable for finding soliton solutions of two practical problems arising in electronics, namely, the conformable time-conformable Generalized Zakharov-Kuznetsov equation (GZKE) and the conformable time-conformable Generalized Zakharov-Kuznetsov-Benjamin-Bona-Mahoney equation (GZK-BBM). The soliton solutions are expressed by two types of functions which are hyperbolic and trigonometric functions.
\end{abstract}

Keywords: Functional variable method; generalized Zakharov-Kuznetsov equation; GZK-BBM equation; conformable derivative.

DOI: https://doi.org/10.31349/RevMexFis.67.050701

\section{Introduction}

Nonlinear partial differential equations (PDEs) are largely used to model various physical events such as shallow water waves, hydrodynamic, physics of plasmas, solid state physics, fluid mechanics, kinematics in chemistry, optic fibers, and chemistry among others. As consequence, the solutions of such equations and, particularly, the traveling wave equation solutions are of interest and become more significant when the impact in these fields is considered. The mathematical theory of these solutions is also significant to support the reliability of the proposed methods. Some recent approaches have begun to draw attention, for example; the $\exp (\varphi(\chi))$-expansion method [1], the modified Khater method [2], the symmetry analysis and fractional logistic function methods [3], the modified exponential expansion approach [4], the Sardar-subequation method [5], the extended rational sine-cosine and rational sinh-cosh methods [6], the Nucci's reduction method [7], simple hyperbolic function ansatzes [8] and so on [9-26].

Here, we plan to employ the method of functional variable to set solutions of the following equations

$$
D_{t}^{\mu} u+\alpha u^{n} u_{x}+\beta\left(u_{x x x}+u_{x y y}\right)=0, \quad n>1,
$$

and

$$
D_{t}^{\mu} u+u_{x}-\alpha\left(u^{n}\right)_{x}+\beta\left(u_{t x x}+u_{x y y}\right)=0, \quad n>1,
$$

where $\alpha, \beta$ and $n$ are arbitrary chosen nonzero constants and $0<\mu \leq 1$. In recent years, some exact solutions for GZKE have been obtained by using different methods; for example, Wazwaz [27] used the extended form of the tangent hyperbolic method to construct solutions to the GZKE describing plasma waves. By the extended type trial equation method, new solitary solutions for the GZKE were obtained in [28]. Mothibi and Khalique [29] used the method based on the simplest equation to report some solutions to the GZKE in new forms. El-Ganaini [30] used the transform defined by ColeHopf with the method of first integral to obtain the complex solutions of the Eq. (1). Deng [31] expressed the solutions of the GZKE in travelling wave forms by the hyperbolic function method in extended form. Furthermore, some exact solutions of the GZK-BBM equation have also been found in some more studies using extended form of the trial equation method, the approach of sine-cosine functions, method of tanh-expansion, and $G^{\prime} / G$-expansion approach [28, 32,33].

In Refs. [34,35], Zerarka et al. defined the method based on functional variable to determine solutions for a many classes PDEs in linear or nonlinear forms. The technique of functional variable has been successfully employed to nonlinear problems. Aminikhah et al., [36] employed it to solve the system of generalized form of the Drinfel'd-SokolovWilson (DSW), Davey-Sterwatson (DS) and Bogoyavlenskii models. Liu and Chen [37] found solutions to the KleinGordon (KG) model, and the Hirota-Satsuma (HS) coupled$\mathrm{KdV}$ model with both time conformable derivatives by using the same method. In another study, the solutions to the general forms of $\mathrm{KG}$ equation, the Camassa-Holm $(\mathrm{CH})$ in 
two space dimensions, the Kadomtsev-Petviashvili and the Schrödinger equations are reported in [38] by the same approach. Eslami et al., [39] applied the method of functional variable to construct the solutions of some problems such as Zakharov model in general aspect, general evolution term $K(m, n)$ model, long-short wave resonance interaction model defined in two space dimensions, and power law nonlinear Schrödinger equation.

Some required definitions of the recently defined derivative, namely Khalil's derivative (KCD), and some significant properties are briefly given below. Significant properties like chain rule or derivative of power functions are also given without proofs. The proofs can be found in the recent related literature.

Definition 1. Suppose $f:(0, \infty) \rightarrow \mathbb{R}$, be a function. Then, $\mathrm{KCD}$ of $f$ of order $\mu$ is expressed

$$
D_{t}^{\mu} f(t)=\lim _{\varepsilon \rightarrow 0} \frac{f\left(t+\varepsilon t^{1-\mu}\right)-f(t)}{\varepsilon},
$$

in the whole positive half space for the order $\mu \in(0,1]$. Some significant properties of the KCD can be summarized below.

Theorem 3. Let $\mu \in(0,1]$, and $f$ and $g$ be differentiable for the order $\mu$ at a point $t$. Then,

(i) $D_{t}^{\mu}(a f(t)+b g(t))=a D_{t}^{\mu} f(t)+b D_{t}^{\mu} g(t), \forall a, b \in \mathbb{R}$.

(ii) $D_{t}^{\mu}\left(t^{\delta}\right)=\mu t^{\delta-\mu}, \forall \mu \in \mathbb{R}$.

(iii) $D_{t}^{\mu}(f(t) g(t))=\left(D_{t}^{\mu} f(t)\right) g(t)+\left(D_{t}^{\mu} g(t)\right) f(t)$.

(iv) $D_{t}^{\mu}\left(\frac{f(t)}{g(t)}\right)=\frac{\left(D_{t}^{\mu} f(t)\right) g(t)-\left(D_{t}^{\mu} g(t)\right) f(t)}{g^{2}(t)}$.

Moreover, if $f$ is differentiable in classical meaning, then the relation is valid $D_{t}^{\mu} f(t)=t^{1-\mu}(d f / d t)$.

In Ref. [8], the rule of chain for the KCD was established.

Theorem 4. Suppose $f:(0, \infty) \rightarrow \mathbb{R}$ be a function satisfying the required conditions to derivative it of order $\mu$ and differentiable in classical meaning and assume that $g$ is another function that satisfies differentiability conditions in classical sense in the range of $f$; then,

$$
D_{t}^{\mu}(f \circ g)(t)=t^{1-\mu} g^{\prime}(t) f^{\prime}(g(t)) \text {. }
$$

\section{The method of functional variable}

Consider the nonlinear time-conformable PDE, whose time derivatives are $\mathrm{KCD}$,

$$
P\left(u, D_{t}^{\mu} u, u_{x}, u_{y}, D_{t}^{2 \mu} u, \ldots\right)=0,
$$

where $u=u(x, y, t)$ solves Eq. (5), the subscripts denote derivatives in partial sense.
The wave transform

$$
\begin{aligned}
u(x, y, t) & =U(\xi), \quad \xi=x+y-c \frac{t^{\mu}}{\mu}, \\
D_{t}^{\mu}(.) & =-c(.)_{\xi}, \quad(.)_{x}=(.)_{\xi}, \\
(.)_{y} & =(.)_{\xi}, \quad D_{t}^{2 \mu}(.)=c^{2}(.)_{\xi \xi},
\end{aligned}
$$

and consequently, the partial differential equation (5) transforms to the ordinal differential equation

$$
G\left(U, U_{\xi}, U_{\xi \xi}, U_{\xi \xi \xi}, \ldots\right)=0 .
$$

Then, assume that the unknown function denoted by capital $U$ is the functional variable satisfying

$$
U_{\xi}=F(U),
$$

and the derivatives of $U$ in various orders are

$$
\begin{aligned}
U_{\xi \xi}= & \frac{1}{2}\left(F^{2}\right)^{\prime}, \\
U_{\xi \xi \xi}= & \frac{1}{2}\left(F^{2}\right)^{\prime \prime} \sqrt{F^{2}}, \\
U_{\xi \xi \xi \xi}= & \frac{1}{2}\left[\left(F^{2}\right)^{\prime \prime \prime} F^{2}+\left(F^{2}\right)^{\prime \prime}\left(F^{2}\right)^{\prime}\right] \\
& \vdots
\end{aligned}
$$

where $F^{\prime}=d F / d U, F^{\prime \prime}=d^{2} F / d U^{2}$ and so on.

The Eq. (7) is an ODE and it can be reduced by employing $U, F$ and its derivatives given in Eq. (9) into Eq. (5) to give

$$
G\left(U, F, F^{\prime}, F^{\prime \prime}, F^{\prime \prime \prime}, \ldots\right)=0 .
$$

This form admits to generate solutions in wide classes covering many traveling waves described by trigonometric or hyperbolic functions. Integrating Eq. (10) gives some new expressions in terms of $F$. Combining Eq. (8) and other relations satisfying required conditions leads to set the solutions of the focused initial problem.

\section{The GZKE with time conformable deriva- tives}

In the present section, we derive solutions in traveling various wave forms of the GZKE with time conformable derivatives by using the method of functional variable described in Sec. 2.

Using the transform $u(x, y, t)=u(\xi)$, where $\xi$ is defined in Eq. (6), the Eq. (1) is transformed to an ODE

$$
-c U+\frac{\alpha}{n+1} U^{n+1}+2 \beta U_{\xi \xi}=0,
$$

or

$$
U_{\xi \xi}=\frac{c}{2 \beta} U-\frac{\alpha}{2 \beta(n+1)} U^{n+1}
$$


Following Eq. (9), one can easily deduce

$$
\frac{1}{2}\left(F^{2}\right)^{\prime}=\frac{c}{2 \beta} U-\frac{\alpha}{2 \beta(n+1)} U^{n+1},
$$

from Eq. (12) . The integral of Eq. (13) with zero integral constant yields

$$
F^{2}=\frac{c}{2 \beta} U^{2}-\frac{\alpha}{\beta(n+1)(n+2)} U^{n+2},
$$

or

$$
F= \pm \sqrt{\frac{c}{2 \beta}} U \sqrt{1-\frac{2 \alpha}{c(n+1)(n+2)} U^{n}} .
$$

From $(8)$ and $(15)$ we find that

$$
\int \frac{d U}{U \sqrt{1-\frac{2 \alpha}{c(n+1)(n+2)} U^{n}}}= \pm \sqrt{\frac{c}{2 \beta}}\left(\xi+\xi_{0}\right),
$$

where $\xi_{0}$ is a constant of integration. Finally, by completing the integration in Eq. (16), two cases of solutions of the timeconformable GZKE after a straightforward algebraic manipulation.

The case $c / \beta>0$ gives the hyperbolic traveling wave solutions $u(x, y, t)$

$$
\begin{aligned}
u_{1,2}(x, y, t) & = \pm\left(\frac{c(n+1)(n+2)}{2 \alpha} \operatorname{sech}^{2}\right. \\
& \left.\times\left[\frac{n}{2} \sqrt{\frac{c}{2 \beta}}\left\{x+y-c \frac{t^{\mu}}{\mu}+\xi_{0}\right\}\right]\right)^{\frac{1}{n}}, \\
u_{3,4}(x, y, t) & = \pm\left(-\frac{c(n+1)(n+2)}{2 \alpha} \operatorname{csch}^{2}\right. \\
& \left.\times\left[\frac{n}{2} \sqrt{\frac{c}{2 \beta}}\left\{x+y-c \frac{t^{\mu}}{\mu}+\xi_{0}\right\}\right]\right)^{\frac{1}{n}} .
\end{aligned}
$$

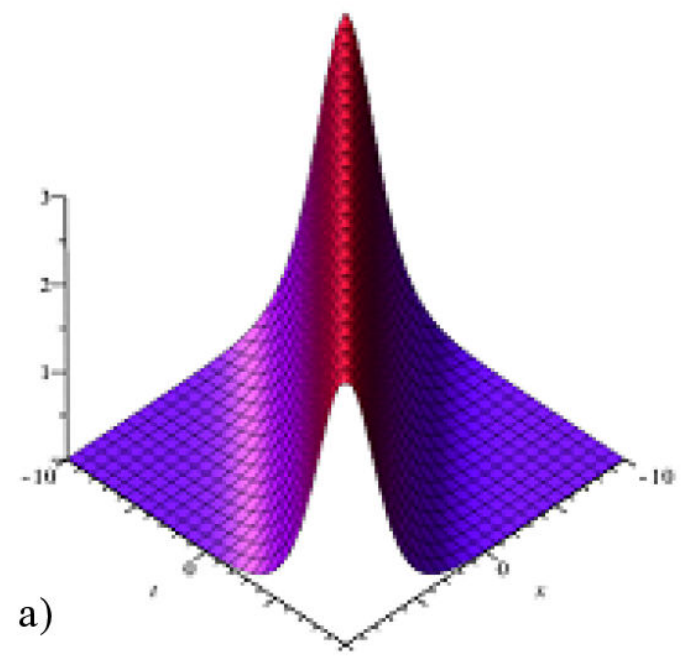

b)
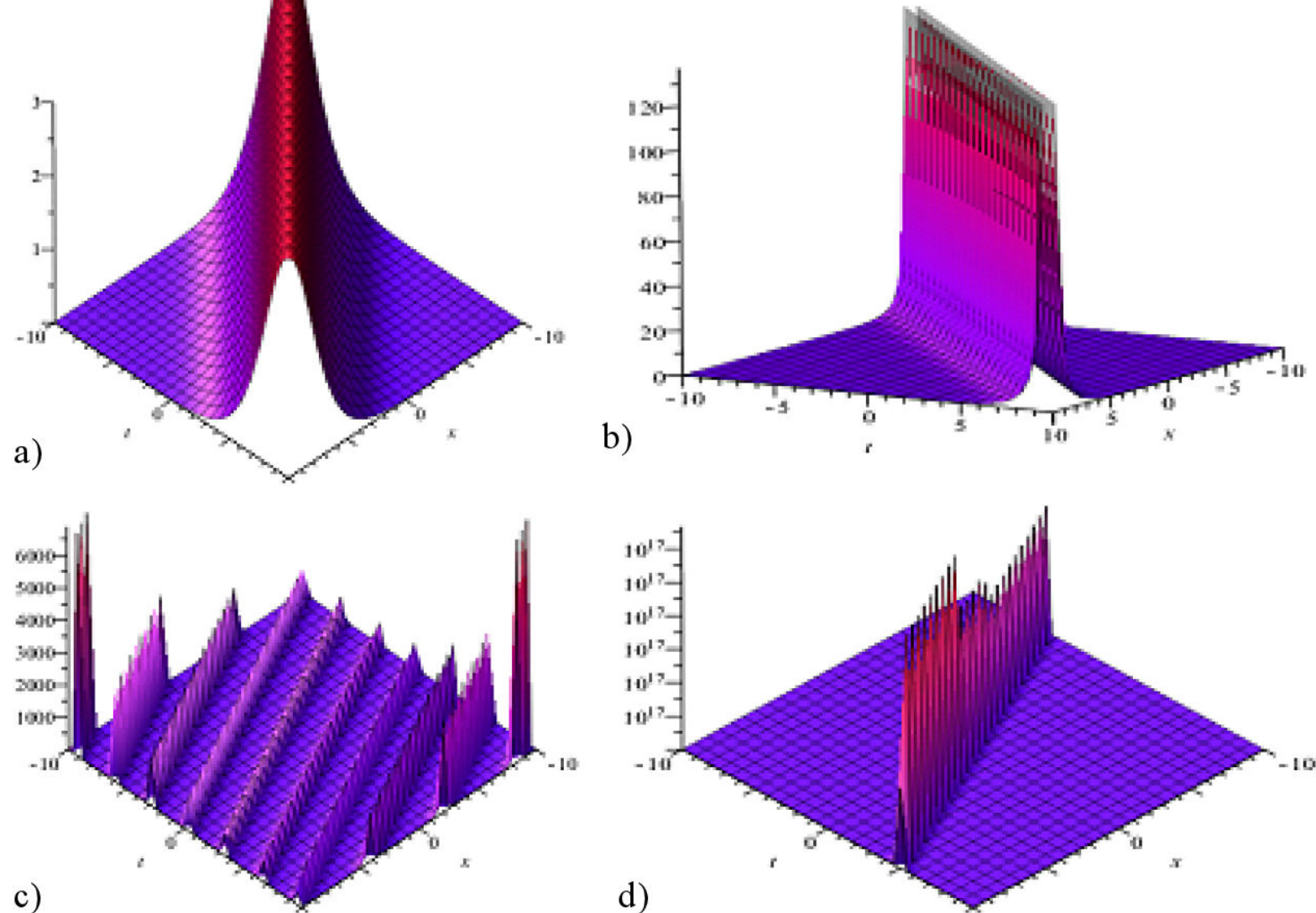

FIGURE 1. The graphical representations of a) bright and b) singular soliton solutions given in Eq. (17) and (18) with the suitably chosen parametric values $n=1, a=1, b=1, c=1, y=0, m=1$ are given respectively. The graphical representations of c) bright and d) singular soliton solutions given in Eq. (19) and (20) with the suitably chosen parametric values $n=2, a=1, b=1, c=2, y=0, m=1$ are. 
The case $c / \beta<0$ gives the periodic traveling wave solutions $u(x, y, t)$

$$
\begin{aligned}
u_{5,6}(x, y, t) & = \pm\left(\frac{c(n+1)(n+2)}{2 \alpha} \sec ^{2}\right. \\
& \left.\times\left[\frac{n}{2} \sqrt{-\frac{c}{2 \beta}}\left\{x+y-c \frac{t^{\mu}}{\mu}+\xi_{0}\right\}\right]\right)^{\frac{1}{n}}, \\
u_{7,8}(x, y, t) & = \pm\left(\frac{c(n+1)(n+2)}{2 \alpha} \csc ^{2}\right. \\
& \left.\times\left[\frac{n}{2} \sqrt{-\frac{c}{2 \beta}}\left\{x+y-c \frac{t^{\mu}}{\mu}+\xi_{0}\right\}\right]\right)^{\frac{1}{n}} .
\end{aligned}
$$

It should be noted that the solutions given in Eq. (17) and Eq. (18) are soliton solutions, in bright and in singular forms, respectively, whilst Eq. (19) and Eq. (20) are singular periodic solutions.

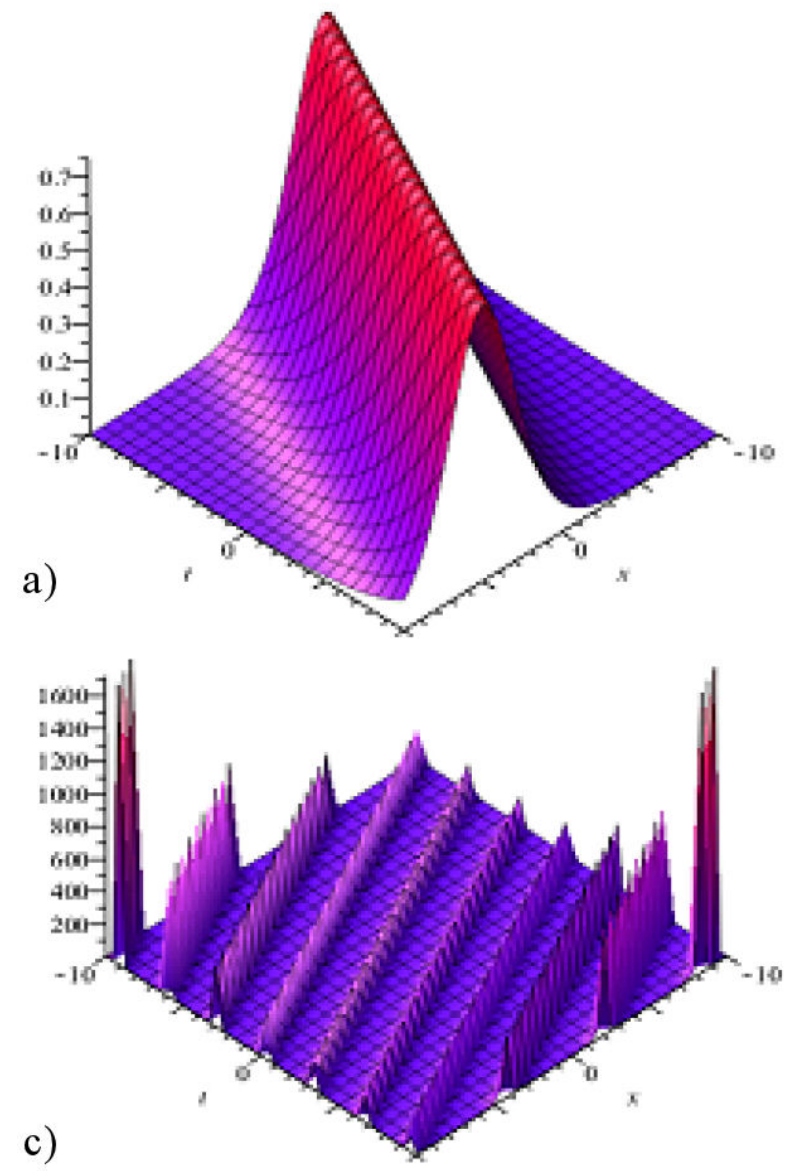

\section{Time-conformable GZK-BBM equation}

In the present Section, we set solutions of the timeconformable GZK-BBM equation by using the method of functional variable described in Sec. 2 .

The two dimensional wave transform $u(x, y, t)=u(\xi)$, where $\xi$ is defined in Eq. (6), the Eq. (7) is

$$
\begin{gathered}
(1-c) U-\alpha U^{n}+\beta(1-c) U_{\xi \xi}=0, \\
U_{\xi \xi}=-\frac{1}{\beta} U-\frac{\alpha}{\beta(c-1)} U^{n} .
\end{gathered}
$$

Following Eq. (9) results from Eq. (22) in an expression for the function $F(U)$

$$
\begin{aligned}
\frac{1}{2}\left(F^{2}\right)^{\prime} & =-\frac{1}{\beta} U-\frac{\alpha}{\beta(c-1)} U^{n}, \\
F^{2} & =-\frac{1}{\beta} U^{2}-\frac{2 \alpha}{\beta(c-1)(n+1)} U^{n+1},
\end{aligned}
$$

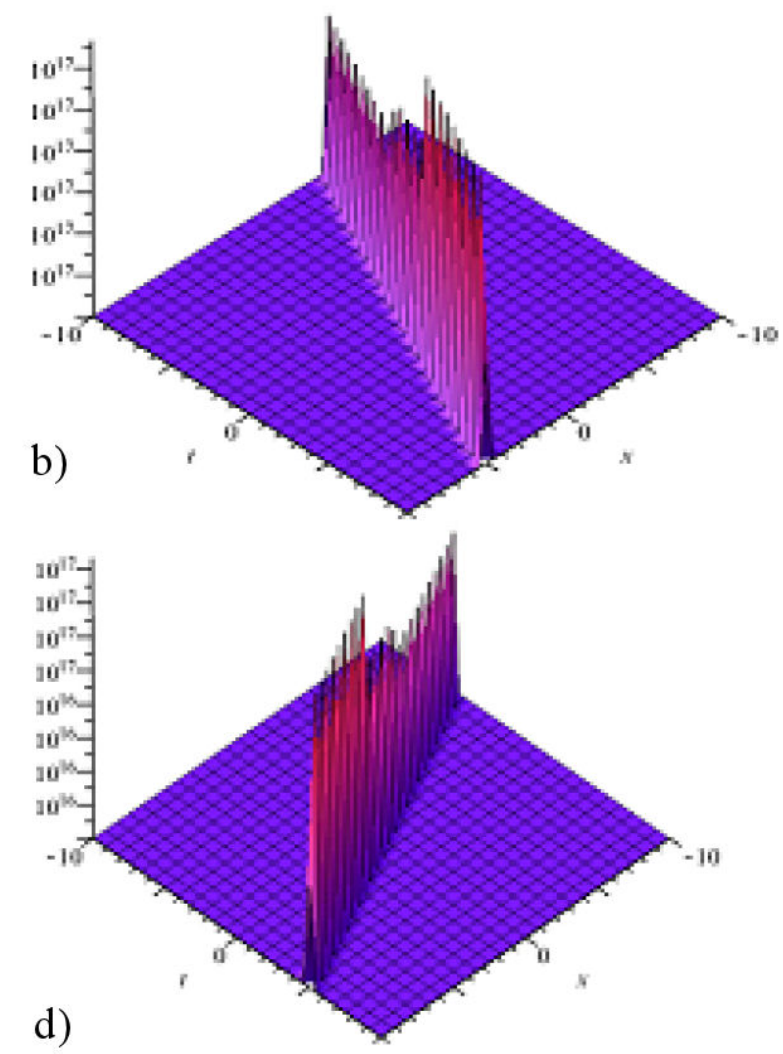

FIgURE 2. The graphical representations of a) bright and b) singular soliton solutions given in Eq. (27) and (28) with the suitably chosen parametric values $n=2, a=1, b=-2, c=0.5, y=0, m=1$ are. The graphical representations of c) bright and d) singular soliton solutions given in Eq. (29) and (30) with the suitably chosen parametric values $n=2, a=1, b=1, c=2, y=0, m=1$ are. 
or

$$
F= \pm \sqrt{-\frac{1}{\beta}} U \sqrt{1+\frac{2 \alpha}{(c-1)(n+1)} U^{n-1}} .
$$

From Eqs. (9) and (26) we deduce that

$$
\int \frac{d U}{U \sqrt{1+\frac{2 \alpha}{(c-1)(n+1)} U^{n-1}}}= \pm \sqrt{-\frac{1}{\beta}}\left(\xi+\xi_{0}\right)
$$

where $\xi_{0}$ is a constant of integration. Finally, by completing the integration in Eq. (26), two cases of travelling wave solutions of the GZK-BBM equation with conformable time derivatives after a straightforward algebraic manipulation.

Case $1 / \beta<0$ gives the hyperbolic traveling wave solutions $u(x, y, t)$

$$
\begin{aligned}
& u_{1,2}(x, y, t)= \pm\left(\frac{(1-c)(n+1)}{2 \alpha} \operatorname{sech}^{2}\right. \\
& \left.\times\left[\frac{n-1}{2} \sqrt{-\frac{1}{\beta}}\left\{x+y-c \frac{t^{\mu}}{\mu}+\xi_{0}\right\}\right]\right)^{\frac{1}{n-1}}, \\
& u_{3,4}(x, y, t)= \pm\left(-\frac{(1-c)(n+1)}{2 \alpha} \operatorname{csch}^{2}\right. \\
& \left.\times\left[\frac{n-1}{2} \sqrt{-\frac{1}{\beta}}\left\{x+y-c \frac{t^{\mu}}{\mu}+\xi_{0}\right\}\right]\right)^{\frac{1}{n-1}},
\end{aligned}
$$

which represent bright and singular soliton solutions, respectively.
Case $1 / \beta>0$ gives the periodic traveling wave solutions $u(x, y, t)$

$$
\begin{gathered}
u_{5,6}(x, y, t)= \pm\left(\frac{(1-c)(n+1)}{2 \alpha} \sec ^{2}\right. \\
\left.\times\left[\frac{n-1}{2} \sqrt{\frac{1}{\beta}}\left\{x+y-c \frac{t^{\mu}}{\mu}+\xi_{0}\right\}\right]\right)^{\frac{1}{n-1}}, \\
u_{7,8}(x, y, t)= \pm\left(\frac{(1-c)(n+1)}{2 \alpha} \csc ^{2}\right. \\
\left.\times\left[\frac{n-1}{2} \sqrt{\frac{1}{\beta}}\left\{x+y-c \frac{t^{\mu}}{\mu}+\xi_{0}\right\}\right]\right)^{\frac{1}{n-1}},
\end{gathered}
$$

which are singular periodic solutions.

\section{Conclusion}

In this paper, the hyperbolic and trigonometric wave-type solutions are constructed for the time conformable forms of the GZK and the time-conformable GZK-BBM equations. The compatible traveling wave transforms defined in two space dimensions reduce the governing equations to some ODEs. Defining a new variable in function form modifies the resulted ODE. The following effort is to solve these ODEs by integration techniques. Due to the good performance of the method of functional variable, it is believed that this method is a promising technique in handling a wide variety of timeconformable PDEs.
1. N. Raza, New optical solitons in nonlinear negative-index materials with Bohm potential. Indian J. Phys. 93 (2019) 657. https://doi.org/10.1007/s12648-018-1234-0

2. M. M. Khater, C. Park, D., Lu, and R. A. Attia, Analytical, semi-analytical, and numerical solutions for the Cahn-Allen equation. Adv. Differ. Equ. 2020 (2020) 9, https://doi. org/10.1186/s13662-019-2475-8.

3. S. Sahoo, S. Saha Ray, M. A. M. Abdou, M. Inc, and Y. M. Chu, New Soliton Solutions of Fractional Jaulent-Miodek System with Symmetry Analysis. Symmetry, 12 (2020) 1001. https://doi.org/10.3390/sym12061001.

4. M. M. Khater, M. Inc, R. A. Attia, D. Lu, and B. Almohsen, Abundant new computational wave solutions of the GM-DP$\mathrm{CH}$ equation via two modified recent computational schemes. J. Taibah Univ. Sci. 14 (2020) 1554, https://doi.org/ $10.1080 / 16583655.2020 .1838137$

5. H. Rezazadeh, M. Inc, and D. Baleanu, New Solitary Wave Solutions for Variants of (3+1)-Dimensional Wazwaz-BenjaminBona-Mahony Equations. Front. Phys. 8 (2020) 332, https : //doi.org/10.3389/fphy.2020.00332

6. J. Vahidi, S. M. Zekavatmand, H. Rezazadeh, M. Inc, M.
A. Akinlar, and Y. M. Chu, New solitary wave solutions to the coupled Maccari's system. Results Phys. 21 (2021) 103801, https://doi.org/10.1016/j.rinp.2020. 103801 .

7. M. S. Hashemi, and A. Akgül, Solitary wave solutions of timespace nonlinear fractional Schrödinger's equation: Two analytical approaches. J. Comput. Appl. Math. 339 (2018) 147, https://doi.org/10.1016/j.cam.2017.11.013

8. A. Korkmaz Exact solutions of space-time fractional EW and modified EW equations. Chaos Solitons Fractals 96 (2017) 132. https://doi.org/10.1016/j.chaos. 2017.01 .015

9. N. Raza, U. Afzal, A. R. Butt, and H. Rezazadeh, Optical solitons in nematic liquid crystals with Kerr and parabolic law nonlinearities. Opt. Quantum Electron. 51 (2019) 107, https: //doi.org/10.1007/s11082-019-1813-0

10. M. S. Hashemi, M. Inc, and A. Yusuf, On three-dimensional variable order time fractional chaotic system with nonsingular kernel. Chaos Solitons Fractals 133 (2020) 109628, https: //doi.org/10.1016/j.chaos.2020.109628. 
11. M. S. Hashemi, Invariant subspaces admitted by fractional differential equations with conformable derivatives. Chaos Solitons Fractals, 107 (2018) 161. https://doi.org/10. $1016 / j . c h a o s .2018 .01 .002$

12. K. Munusamy, C. Ravichandran, K. S. Nisar, and B. Ghanbari, Existence of solutions for some functional integrodifferential equations with nonlocal conditions. Math. Methods Appl. Sci. 43 (2020) 10319, https://doi.org/10. $1002 / \mathrm{mma} .6698$

13. G. Rahman, K. S. Nisar, B. Ghanbari, and T. Abdeljawad, On generalized fractional integral inequalities for the monotone weighted Chebyshev functionals. Adv. Differ. Equ. 2020 (2020) 368, https://doi.org/10.1186/ s13662-020-02830-7

14. B. Ghanbari, A. Yusuf, and D. Baleanu, The new exact solitary wave solutions and stability analysis for the (2+1)-dimensional Zakharov-Kuznetsov equation. Adv. Differ. Equ. 2019 (2019) 49, https://doi.org/10.1186/ s13662-019-1964-0.

15. B. Ghanbari, K. S. Nisar, and M. Aldhaifallah, Abundant solitary wave solutions to an extended nonlinear Schrödinger's equation with conformable derivative using an efficient integration method. Adv. Differ. Equ. 2020 (2020) 328, https: //doi.org/10.1186/s13662-020-02787-7

16. L. Akinyemi, M. Şenol, and O. S. Iyiola, Exact solutions of the generalized multidimensional mathematical physics models via sub-equation method. Comput. Simul. 182 (2021) 211, https://doi.org/10.1016/j.matcom.2020. 10.017 .

17. M. Şenol, O. S. Iyiola, H. D. Kasmaei, and L. Akinyemi, Efficient analytical techniques for solving time-fractional nonlinear coupled Jaulent-Miodek system with energy-dependent Schrödinger potential. Adv. Differ. Equ. 2019 (2019) 462, https://doi.org/10.1186/s13662-019-2397-5.

18. L. Akinyemi, A fractional analysis of Noyes-Field model for the nonlinear Belousov-Zhabotinsky reaction. Comput. Appl. Math. 39 (2020) 175, https://doi.org/10.1007/ S40314-020-01212-9

19. L. Akinyemi, M. Şenol, and S. N. Huseen, Modified homotopy methods for generalized fractional perturbed Zakharov-Kuznetsov equation in dusty plasma. Adv. Differ. Equ. 2021 (2021) 45, https://doi.org/10.1186/ s13662-020-03208-5

20. M. Munawar, A. Jhangeer, A. Pervaiz, and F. Ibraheem, New general extended direct algebraic approach for optical solitons of Biswas-Arshed equation through birefringent fibers. $O p$ tik 228 (2020) 165790. https://doi.org/10.1016/j. ijleo.2020.165790

21. A. Jhangeer, A. Hussain, M. Junaid-U-Rehman, D. Baleanu, and M. B. Riaz, Quasi-periodic, chaotic and travelling wave structures of modified Gardner equation. Chaos, Solitons and Fractals, 143 (2020) 110578, https : / / doi.org/10. $1016 / j$.chaos.2020.110578

22. A. Hussain, A. Jhangeer, N. Abbas, I. Khan, and E. S. M. Sherif, Optical solitons of fractional complex Ginzburg-Landau equation with conformable, beta, and Mtruncated derivatives: a comparative study. Adv. Differ.
Equ. 2020 (2020) 612, https://doi.org/10.1186/ s13662-020-03052-7

23. E. C. Aslan, and M. Inc, Optical soliton solutions of the NLSE with quadratic-cubic-Hamiltonian perturbations and modulation instability analysis. Optik 196 (2019) 162661, https: //doi.org/10.1016/j.ijleo.2019.04.008

24. A. Yusuf, M. Inc, A. I. Aliyu, and D. Baleanu, Optical solitons possessing beta derivative of the Chen-Lee-Liu equation in optical fiber. Front. Phys. 7 (2019) 34, https://doi.org/ $10.3389 /$ fphy.2019.00034

25. R. Abazari, Application of extended tanh function method on KdV-Burgers equation with forcing term. Rom. J. Phys, 59 (2014) 3.

26. A. Kurt, New Analytical and Numerical Results For Fractional Bogoyavlensky-Konopelchenko Equation Arising in Fluid Dynamics. Appl. Math. 35 (2020) 101, https://doi.org/ $10.1007 / \mathrm{s} 11766-020-3808-9$

27. A. M. Wazwaz, The extended tanh method for the ZakharovKuznetsov (ZK) equation, the modified ZK equation, and its generalized forms. Commun. Nonlinear Sci. Numer. Simul. 13 (2008) 1039, https://doi.org/10.1016/j.cnsns. 2006.10 .007

28. S. T. Mohyud-Din, and A. Irshad, Solitary wave solutions of some non linear PDEs arising in electronics. Opt. Quantum Electron. 49 (2017) 130, https://doi.org/10.1007/ s11082-017-0974-y

29. D. M. Mothibi, and C. M. Khalique, Conservation laws and exact solutions of a generalized Zakharov-Kuznetsov equation. Symmetry 7 (2015) 949, https://doi.org/10.3390/ sym7020949

30. S. I. El-Ganaini, Travelling Wave Solutions of the ZakharovKuznetsov Equation in Plasmas with Power Law Non linearity. Int. J. Contemp. Math. Sci., 6 (2011) 2353.

31. C. Deng, New exact solutions to the Zakharov-Kuznetsov equation and its generalized form. Commun. Nonlinear Sci. Numer. Simul. 15 (2010) 857, https://doi.org/10.1016/j. Cnsns.2009.05.011

32. A. M. Wazwaz, Compact and noncompact physical structures for the ZK-BBM equation. Appl. Math. Comput. 169 (2005) 713, https://doi.org/10.1016/j.amc.2004.09. 062

33. K. R. Adem, and C. M. Khalique, Conservation Laws and Traveling Wave Solutions of a Generalized Nonlinear ZK-BBM Equation, Abstr. Appl. Anal. 2014 (2014) 139513, https: //doi.org/10.1155/2014/139513

34. A. Zerarka, S. Ouamane, and A. Attaf, On the method of functional variable for finding exact solutions to a class of wave equations. Appl. Math. Comput. 217 (2010) 2897, https: //doi.org/10.1016/j.amc.2010.08.070

35. A. Zerarka, and S. Ouamane, Application of the method of functional variable to a class of non linear wave equations. World J. Model. Simul. 6 (2010) 150.

36. H. Aminikhaha, A. R. Sheikhani, and H. Rezazadeh, Exact solutions of some non linear systems of partial differential equations by using the method of functional variable. Mathematica, 56 (2014) 103. 
37. W. Liu, and K. Chen, The method of functional variable for finding exact solutions of some non linear time-fractional differential equations. Pramana, 81 (2013) 377. https : / / doi. org/10.1007/s12043-013-0583-7.

38. A. Nazarzadeh, M. Eslami, and M. Mirzazadeh, Exact solutions of some non linear partial differential equations using functional variable method. Pramana, 81 (2013) 225. https : //doi.org/10.1007/s12043-013-0565-9.
39. M. Eslami, and M. Mirzazadeh, Functional variable method to study non linear evolution equations. Open Eng. 3 (2013) 451. https://doi.org/10.2478/s13531-013-0104-y

40. R. Khalil, M. Al Horani, A. Yousef, and M. Sababheh, A new definition of fractional derivative, J. Comput. Appl. Math. 264 (2014) 65, https://doi.org/10.1016/j.cam. 2014.01 .002 